Article

\title{
Electrochemical Properties of Poly(Anthraquinonyl Sulfide)/Graphene Sheets Composites as Electrode Materials for Electrochemical Capacitors
}

\section{Wonkyun Lee ${ }^{1, *}$, Shinya Suzuki ${ }^{1}$ and Masaru Miyayama ${ }^{1,2}$}

1 School of Engineering, The University of Tokyo, 7-3-1 Hongo, Bunkyo-ku, Tokyo 113-8656, Japan; E-Mails: sin@fmat.t.u-tokyo.ac.jp (S.S.); miyayama@fmat.t.u-tokyo.ac.jp (M.M.)

2 CREST, Japan Science and Technology Agency, 4-8-1 Honcho, Kawaguchi, Saitama 332-0012, Japan

* Author to whom correspondence should be addressed; E-Mail: wklee@fmat.t.u-tokyo.ac.jp; Tel.: +81-3-5841-7642; Fax: +81-3-5841-0250.

Received: 14 April 2014; in revised form: 30 June 2014 / Accepted: 23 July 2014 /

Published: 30 July 2014

\begin{abstract}
Poly(anthraquinonyl sulfide) (PAQS)/graphene sheets (GSs) composite was synthesized through in situ polymerization to evaluate its performance as an electrode material for electrochemical capacitors. PAQS was successfully synthesized in the presence of GSs with uniform distribution. PAQS/GSs showed a pair of reversible redox peaks at around $0 \mathrm{~V}(v s . \mathrm{Ag} / \mathrm{AgCl})$. The specific capacitance of PAQS/GSs was $349 \mathrm{~F} \cdot \mathrm{g}^{-1}$ $\left(86 \mathrm{mAh} \cdot \mathrm{g}^{-1}\right)$ at a current density of $500 \mathrm{~mA} \cdot \mathrm{g}^{-1}$, and a capacitance of $305 \mathrm{~F} \cdot \mathrm{g}^{-1}$ was maintained even at a high current density of $5000 \mathrm{~mA} \cdot \mathrm{g}^{-1}$. The in situ polymerization of PAQS with GSs facilitated their interaction and enabled faster charge transfer and redox reaction, resulting in enhanced electrode properties.
\end{abstract}

Keywords: Poly(anthraquinonyl sulfide); graphene; composite; electrochemical capacitors

\section{Introduction}

The increased demand for clean, renewable energy has led to intense research efforts into energy storage systems. Electrochemical capacitors (ECs) are of particular interest due to their high power density and long cycle life [1-4]. In particular, ECs using aqueous instead of organic electrolyte have the additional advantage of high safety. ECs can store energy via two mechanisms: electrostatic charge 
separation at the electrode/electrolyte interface, or Faradaic redox reaction at the surface of the active electrode material $[5,6]$. Recent studies have focused on improving the limited energy density of ECs by exploring high-capacitance electrode materials such as transition-metal oxides [7-9] and conducting polymers [10-12].

Quinone-based materials have attracted considerable attention as electrode materials for energy storage devices such as lithium-ion batteries [13-17], redox flow batteries [18], polymer/air batteries [19] and electrochemical capacitors [20-22]. Although quinone-based materials can provide high capacitance through a two-electron redox reaction, degradation of performance due to the dissolution of quinone molecules into both aqueous and organic electrolytes is a significant obstacle to their use in energy storage devices [14,21,22]. Recently, quinone polymer electrodes such as poly(2,5-dihydroxy1,4-benzoquinone-3,6-methylene) (PDBM) [23], poly(2,5-dihydroxy-1,4-benzoquinonyl sulfide) (PDBS) [24] and poly(anthraquinonyl sulfide) (PAQS) [13,25] exhibited enhanced cycling stability with relatively high capacitance in lithium-ion batteries. Among these, PAQS was synthesized by a simple method and showed high reversible capacitance of $185 \mathrm{mAh} \cdot \mathrm{g}^{-1}$ with good cyclability [13]. In the present study, we focused on PAQS as an electrode material for electrochemical capacitors. To the best of our knowledge, there are no reports on PAQS electrodes using aqueous electrolytes.

Graphene sheets (GSs) are single-atom-thick carbon sheets with outstanding properties that include large specific surface area [26,27], high electrical conductivity [28] and excellent mechanical performance [29]. Previous attempts to produce GSs composites with redox-active polymers resulted in composites that showed good performance due to the enhanced electrical conductivity of the polymers in combination with graphene [30-32].

Here, we synthesized PAQS in the presence of dispersed GSs (PAQS/GSs) to investigate the influence of in situ polymerization of PAQS with GSs on the electrode properties for electrochemical capacitors. This in situ polymerization was a simple process because of the favorable $\pi-\pi$ interactions between PAQS and GSs. The microstructure and electrode properties of PAQS/GSs were evaluated in order to understand the reaction activity of PAQS in aqueous electrolyte and the effect of in situ polymerization with GSs.

\section{Results and Discussion}

\subsection{FT-IR Spectra of GSs, PAQS and PAQS/GSs}

Figure 1 shows the FT-IR spectra of GSs, PAQS and PAQS/GSs. As shown in Figure 1a, the featureless FT-IR spectrum of GSs indicates that the peaks for the oxygen functional groups were almost completely removed by thermal exfoliation [33]. The FT-IR spectrum of PAQS shows absorbance peaks that are consistent with those of reported PAQS [13,25,34] (Figure 1b). The peaks at 1676 and $1568 \mathrm{~cm}^{-1}$ represent, respectively, $\mathrm{C}=\mathrm{O}$ and $\mathrm{C}=\mathrm{C}$ stretching vibrations of the anthraquinonyl group. The peaks at 1413 and $1129 \mathrm{~cm}^{-1}$ correspond to the stretching of the sulfur-disubstituted aromatic ring and the ring-sulfur, respectively. These results indicate that anthraquinone-based PAQS was successfully synthesized. As shown in Figure 1c, the absorbance peaks of PAQS/GSs were similar to those of PAQS, indicating that PAQS is well synthesized even in the presence of GSs. 
Figure 1. FT-IR spectra of (a) graphene sheets (GSs); (b) poly(anthraquinonyl sulfide) (PAQS); and (c) PAQS/GSs.

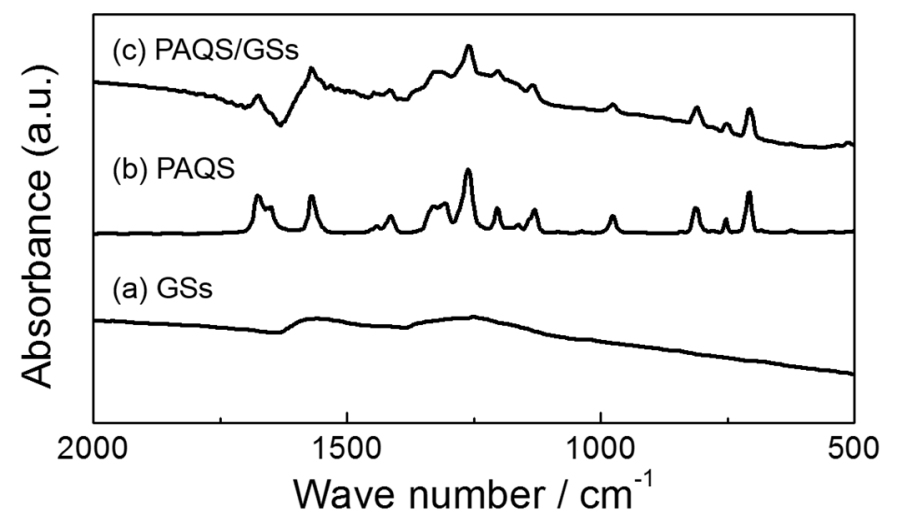

\subsection{Raman Spectra for GSs, $P A Q S$ and $P A Q S / G S S$}

Figure 2 shows the results of Raman spectra for GSs, PAQS and PAQS/GSs. In the GSs, two bands were observed at about 1340 and $1580 \mathrm{~cm}^{-1}$, corresponding to the $\mathrm{G}$ band and D band (Figure 2a). The $\mathrm{G}$ band is associated with the ordered $\mathrm{sp} 2$ carbon lattice and the $\mathrm{D}$ band originates from the structural defects and disorders in the graphene layer $[35,36]$. For PAQS, in-plane $\mathrm{C}-\mathrm{H}$ bending vibrations and symmetric stretching of sulfur-disubstituted ring, and $\mathrm{C}=\mathrm{O}$ stretching vibrations of the anthraquinonyl group appeared at 1177, 1570 and $1642 \mathrm{~cm}^{-1}$, respectively [37,38] (Figure 2b). For PAQS/GSs, two dominant peaks were observed similarly to GSs (Figure 2c). PAQS/GSs presents a shift in the G band towards lower wave numbers compared to GSs due to the $\pi-\pi$ interactions between the polymer and graphene sheets, indicating that PAQS is well combined with the GSs [31,39]. The disappearance of in-plane $\mathrm{C}-\mathrm{H}$ bending and $\mathrm{C}=\mathrm{O}$ stretching vibrations of PAQS is another evidence of the $\pi-\pi$ interactions [39]. The relatively weak Raman signal for PAQS is similar to that of highly dispersed polymers on graphene [40,41], which reveals that PAQS is well dispersed on the graphene sheets.

Figure 2. Raman spectra of (a) GSs; (b) PAQS; and (c) PAQS/GSs at an excitation wavelength of $514 \mathrm{~nm}$.

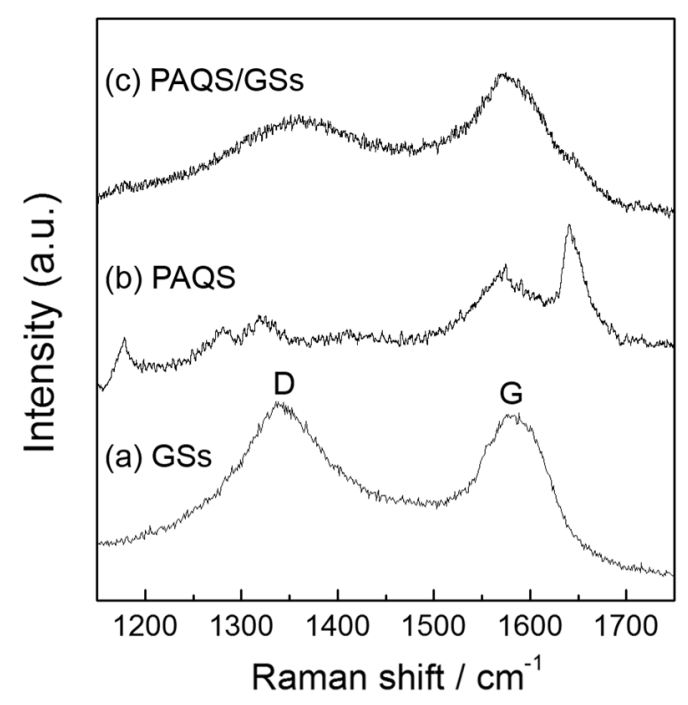




\subsection{XRD Patterns for GSs, PAQS and PAQS/GSs}

Figure 3 shows the XRD patterns for GSs, PAQS and PAQS/GSs. As can be seen in Figure 3a, GSs exhibited a broad diffraction peak at around $2^{\circ}-21^{\circ}$, indicating that graphene sheets are randomly stacked compared to graphite. For PAQS, the crystalline peaks appeared at $12^{\circ}, 22^{\circ}$ and $24^{\circ}$, which is consistent with previously reported data [13] (Figure 3b). The XRD patterns of PAQS/GSs exhibit one dominant diffraction peak similar to that of GSs but with the main peak position at $24^{\circ}$, indicating that the weak diffraction peak of PAQS is combined with that of GSs (Figure 3c). The results of FT-IR, Raman and XRD suggest that the synthesis of a homogeneous PAQS/GSs composite was successful.

Figure 3. XRD patterns of (a) GSs; (b) PAQS and (c) PAQS/GSs.

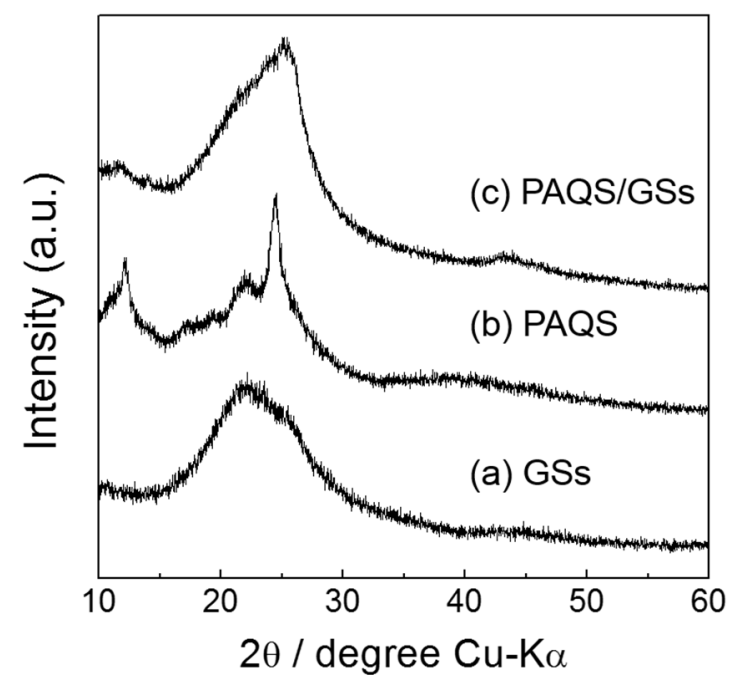

\section{4. $N_{2}$ Adsorption-Desorption Isotherms of GSs, PAQS, PAQS/GSs(m) and PAQS/GSs}

To investigate the influence of in situ polymerization of PAQS with GSs on the electrode properties for electrochemical capacitors, we prepared and measured mechanically mixed PAQS/GSs $(\mathrm{m})$ as a comparison material. Figure 4 shows the $\mathrm{N}_{2}$ adsorption-desorption isotherms of GSs, PAQS, PAQS/GSs(m) and PAQS/GSs. In the GSs, obvious hysteresis loop was observed at relative pressure ranging from 0.45 to 1.0 , which is attributed to a mesoporous structure (Figure 4a). GSs exhibited a large BET surface area of $849 \mathrm{~m}^{2} \cdot \mathrm{g}^{-1}$. As shown in Figure $4 \mathrm{~b}$, however, PAQS showed a BET surface area of $27 \mathrm{~m}^{2} \cdot \mathrm{g}^{-1}$, which is attributed to the aggregation of polymers during the polymerization process. In the PAQS/GSs $(\mathrm{m})$, a hysteresis loop which is originated from the structure of GSs was still observed, indicating that significant aggregation was not accompanied during mechanical mixing process (Figure 4c). PAQS/GSs $\left(\mathrm{m}\right.$ ) exhibited a BET surface area of $190 \mathrm{~m}^{2} \cdot \mathrm{g}^{-1}$. As shown in Figure 4d, PAQS/GSs exhibited a decreased specific surface area of $42 \mathrm{~m}^{2} \cdot \mathrm{g}^{-1}$ compared to GSs, and this is greatly attributed to the enhanced aggregation of the PAQS/GSs particles during the in situ polymerization. 
Figure 4. $\mathrm{N}_{2}$ adsorption-desorption isotherms for (a) GSs; (b) PAQS; (c) PAQS/GSs(m); and (d) PAQS/GSs.
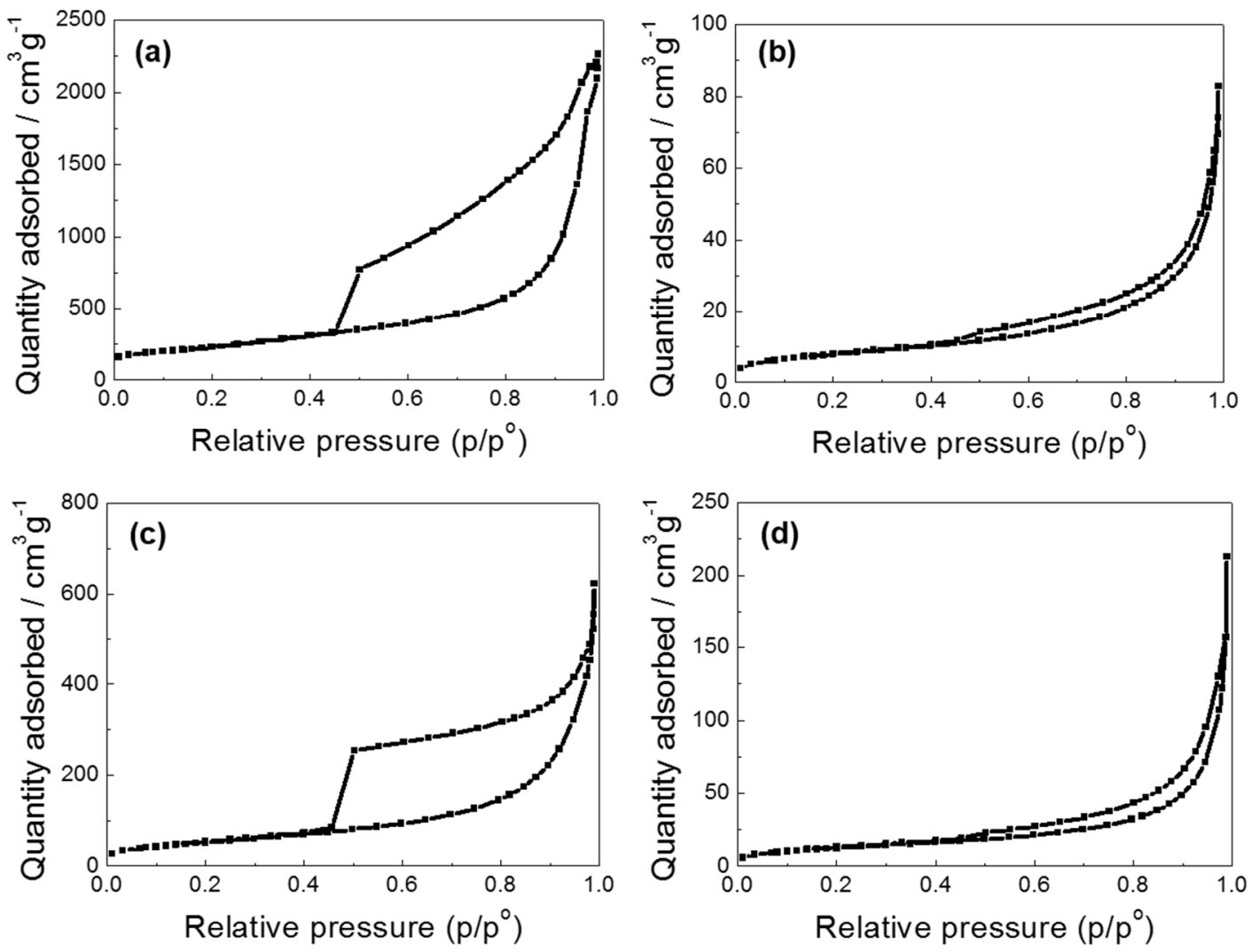

\subsection{Microstructures of GSs, PAQS, PAQS/GSs $(m)$ and PAQS/GSs}

Figure 5 illustrates the microstructures and morphology of GSs, PAQS, PAQS/GSs $(\mathrm{m})$ and PAQS/GSs. As shown in Figure 5a, GSs has a wrinkled morphology, which is attributed to the insertion and removal of oxygen functional groups during the preparation process, and the graphene sheets were loosely agglomerated. The pure PAQS exhibits firmly aggregated structure with a size of 1-5 $\mu \mathrm{m}$ (Figure 5b). As shown in Figure 5c, there is no aggregation of PAQS particles in the PAQS/GS, and a smooth surface and increased thickness of sheets were observed. This reveals that the surface of GSs is covered with highly dispersed PAQS due to the favorable $\pi-\pi$ interactions between PAQS and GSs [30]. A TEM image shows that the PAQS/GSs sheets are nearly transparent under electron irradiation, implying that PAQS is dispersed on graphene sheets with extremely thin morphology (Figure 5d). In the PAQS/GS(m), however, PAQS was not fully dispersed due to the recurrence of aggregation during the preparation process (Figure 5e). Energy-dispersive X-ray (EDX) spectroscopy elemental mapping was used to investigate the distribution of PAQS in the composite and mixture. The elemental mapping of sulfur on PAQS/GSs $(\mathrm{m})$ shown in Figure $5 \mathrm{f}$ reveals the aggregated PAQS in the structure. In the case of PAQS/GSs, SEM images and elemental mapping of sulfur reveal that PAQS is uniformly dispersed on the GSs (Figure $5 \mathrm{~g}, \mathrm{~h}$ ). 
Figure 5. SEM images of (a) GSs; (b) PAQS; and (c) PAQS/GSs; (d) TEM image of PAQS/GSs. SEM images and energy-dispersive X-ray (EDX) sulfur elemental mapping images of (e,f) PAQS/GS(m) and (g,h) PAQS/GS.
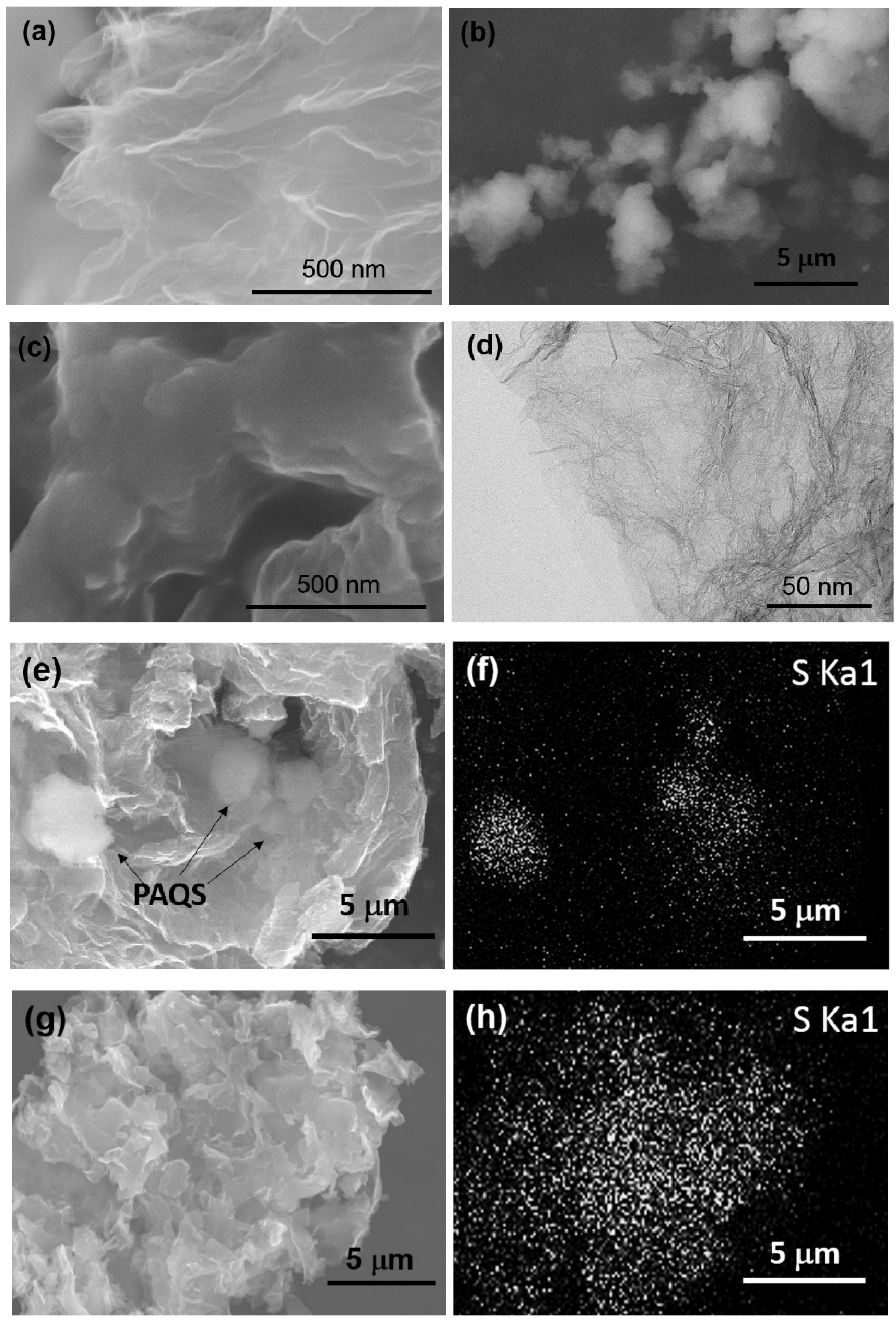


\subsection{Electrochemical Properties}

\subsubsection{Cyclic Voltammograms}

Cyclic voltammetry (CV) curves after 200 cycles for GSs, PAQS/GSs(m) and PAQS/GSs at a scan rate of $0.5 \mathrm{mV} \cdot \mathrm{s}^{-1}$ between -0.2 and $0.8 \mathrm{~V}(v s . \mathrm{Ag} / \mathrm{AgCl})$, which is the electrochemically stable potential window in $0.5 \mathrm{M} \mathrm{H}_{2} \mathrm{SO}_{4}$ electrolyte, are shown in Figure 6a. The CV curve for GSs shows a typical rectangular-like voltammogram without obvious redox peaks, indicating that the electric double-layer capacitance contributes dominantly to the capacitance of GSs. For PAQS/GSs(m), a pair of weak peaks was observed in the range of 0 to $-0.2 \mathrm{~V}$ ( $v s . \mathrm{Ag} / \mathrm{AgCl})$, implying that PAQS has redox activity in aqueous electrolyte. PAQS/GSs showed a pair of strong redox peaks at around $0 \mathrm{~V}$ (vs. $\mathrm{Ag} / \mathrm{AgCl}$ ), which are possibly attributed to the original redox characteristics of PAQS in $0.5 \mathrm{M}$ $\mathrm{H}_{2} \mathrm{SO}_{4}$ electrolyte. The anthraquinonyl groups of PAQS are known to have redox activity through electrochemical association with various cations, such as $\mathrm{Li}^{+}$and $\mathrm{Na}^{+}[13,34]$. Thus, it is suggested that the redox mechanism of PAQS could be similar to that in organic electrolytes. Scheme I shows a possible redox mechanism of PAQS in aqueous electrolytes, and its theoretical capacitance is expected to be $225 \mathrm{mAh}$ (g-PAQS) ${ }^{-1}$. The delocalization of electrons over the entire molecule is assumed to be more favorable in PAQS than that of anthraquinone due to the enlarged $\pi$-system, and this could bring out the redox peaks shift to more positive potential [17,42] than that of anthraquinone in aqueous solution $(-0.16 \mathrm{~V}(v s . \mathrm{Ag} / \mathrm{AgCl})[22])$. Therefore, the newly appearing redox peaks at around $0 \mathrm{~V}$ (vs. $\mathrm{Ag} / \mathrm{AgCl}$ ) in the present study are attributed to the original redox reaction activity of PAQS. Furthermore, it is suggested that the distribution of PAQS is an important factor of the redox reaction in aqueous solution because PAQS that was not fully dispersed showed very low reaction activity.

Figure 6. (a) Cyclic voltammograms at a scan rate of $0.5 \mathrm{mV} \cdot \mathrm{s}^{-1}$ after 200 cycles; and (b) comparison of capacitance values with cycle number for GSs, PAQS/GSs(m) and PAQS/GSs in $0.5 \mathrm{M} \mathrm{H}_{2} \mathrm{SO}_{4}$.
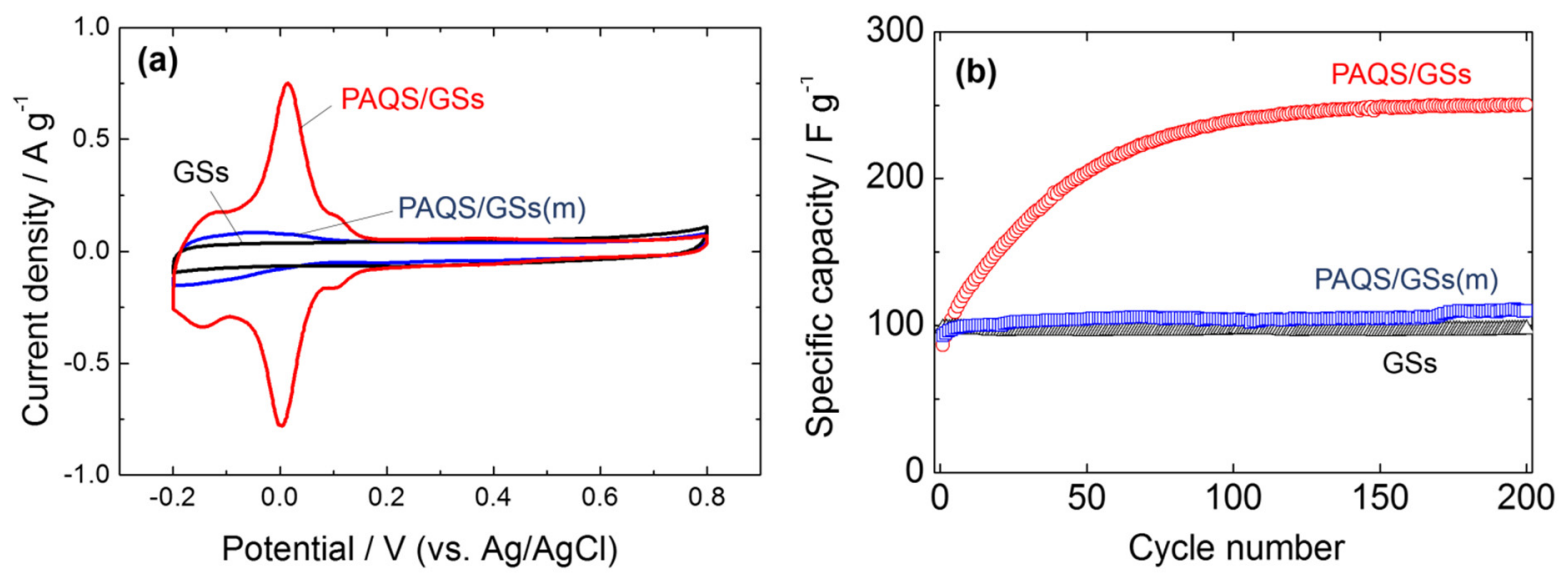

Figure $6 \mathrm{~b}$ shows the specific capacitance of GSs, PAQS/GSs $(\mathrm{m})$ and PAQS/GSs calculated from their CV curves and normalized by the electrode weight. GSs and PAQS/GSs(m) showed relatively good cycling stability and exhibited capacitance of 98 and $110 \mathrm{~F} \cdot \mathrm{g}^{-1}$ (corresponding to 27 and $31 \mathrm{mAh} \cdot \mathrm{g}^{-1}$ ) at the 200th cycle, respectively. In the PAQS/GSs, the specific capacitance increases in 
the initial stage and then maintains stable capacitance of $250 \mathrm{~F} \cdot \mathrm{g}^{-1}\left(70 \mathrm{mAh} \cdot \mathrm{g}^{-1}\right)$. This type of activation process can be seen in conducting polymer electrodes, a phenomenon reportedly related to the gradual formation of electronic conducting pathways during redox reactions [23,43-45]. Thus, it is suggested that the PAQS/GSs electrode is almost activated and reaches the optimum condition.

Scheme I. Electrochemical redox mechanism of PAQS in $0.5 \mathrm{M} \mathrm{H}_{2} \mathrm{SO}_{4}$ aqueous solution.
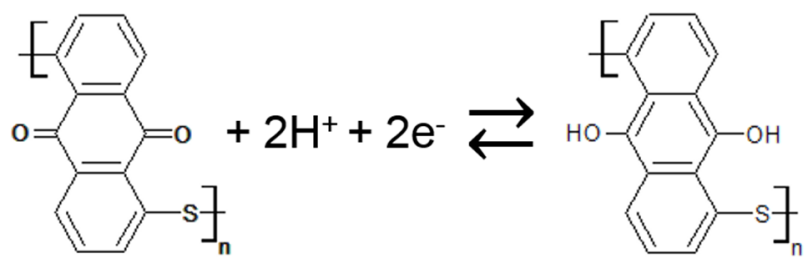

PAQS

\subsubsection{Rate Capabilities}

Figure 7a shows the constant-current charge/discharge curves for PAQS/GSs between $-0.1 \mathrm{~V}$ and $0.8 \mathrm{~V}$ (vs. $\mathrm{Ag} / \mathrm{AgCl}$ ) in $0.5 \mathrm{M} \mathrm{H}_{2} \mathrm{SO}_{4}$ at different current densities. The charge/discharge tests were carried out on the fully activated PAQS/GSs electrode. The charge/discharge curves of PAQS/GSs showed a symmetrical shape with a potential plateau corresponding to the reaction of PAQS, and the specific capacitance of PAQS/GSs at a current density of $500 \mathrm{~mA} \cdot \mathrm{g}^{-1}$ was $349 \mathrm{~F} \cdot \mathrm{g}^{-1}\left(86 \mathrm{mAh} \cdot \mathrm{g}^{-1}\right)$.

Figure 7. (a) Charge/discharge curves at different current densities; and (b) plots of specific capacitance versus discharge current density for PAQS/GSs.
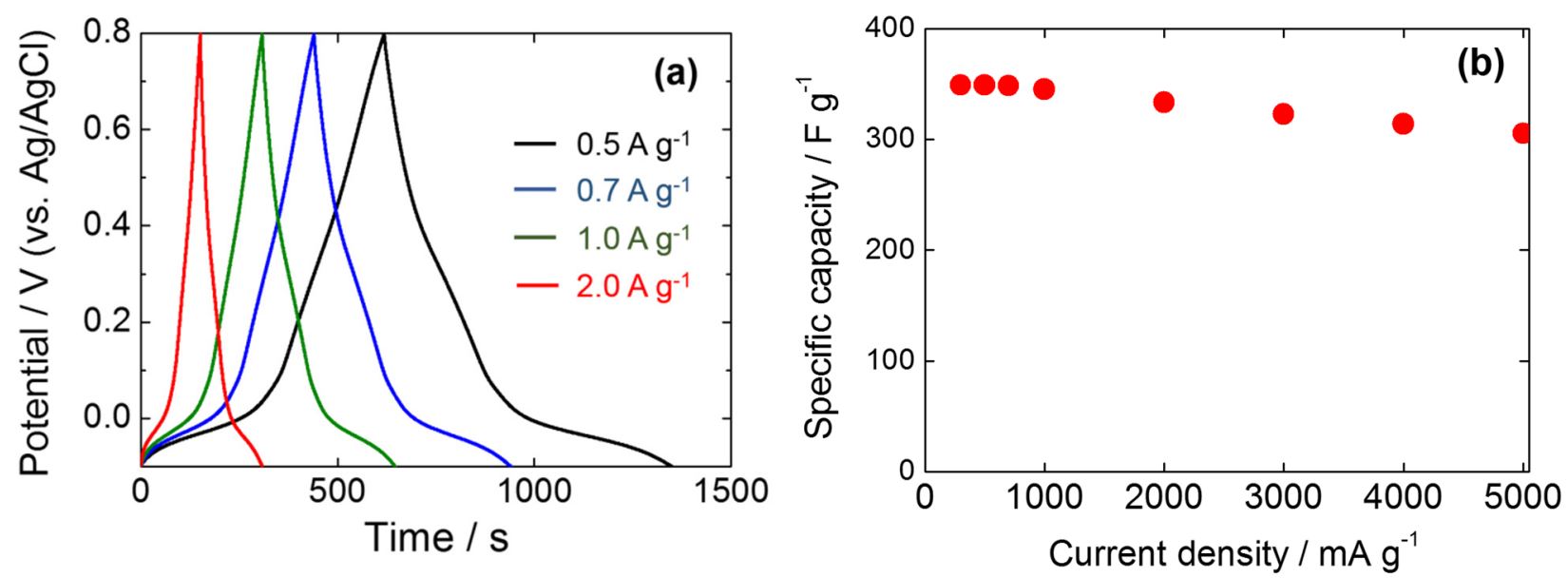

From the $\mathrm{CV}$ and charge/discharge curves for PAQS/GSs, it is postulated that redox capacitance of PAQS is generated at the potential region lower than $0.2 \mathrm{~V}(v s . \mathrm{Ag} / \mathrm{AgCl})$. The calculated specific capacitance of PAQS corresponds to $156 \mathrm{mAh}$ (g-PAQS) ${ }^{-1}$, which is almost $70 \%$ of the theoretical capacitance of PAQS. Charge/discharge curves almost maintain the same shape even at a high current density of $2000 \mathrm{~mA} \cdot \mathrm{g}^{-1}$. This indicates that PAQS/GSs has electrochemically reversible charge-discharge properties. As shown in Figure 7b, the specific capacitance of PAQS/GSs at a high current density of $5000 \mathrm{~mA} \cdot \mathrm{g}^{-1}$ is $305 \mathrm{~F} \cdot \mathrm{g}^{-1}$. This good rate capability of PAQS/GSs seems to have 
resulted from enhanced electrical conductivity corresponding to the influence of the GSs. Song et al. [30] reported that even by mixing a small amount of graphene with polymers, conductivities are greatly improved. This indicates that the electrochemical performance of PAQS/GSs is improved compared with pristine PAQS due to the synergistic effect between GSs and PAQS. The capacitance of PAQS/GSs decreased by only $2 \%$ even after 500 charging/discharging cycles at a current density of $500 \mathrm{~mA} \cdot \mathrm{g}^{-1}$, indicating that PAQS/GSs has long-term electrochemical stability. In the case of PAQS/GSs $(\mathrm{m})$, the capacitance increased by $1 \%$ after 500 cycles, which reveals the very slow activation process of PAQS. Based on these results, in situ polymerization of PAQS in the presence of GSs is found to be effective for improving the electrode properties for use in electrochemical capacitors using aqueous electrolyte solutions.

\section{Experimental Section}

\subsection{Material Synthesis}

PAQS was prepared through the polycondensation of 1,5-dichloroanthraquinone (15DCAQ) and anhydrous sodium sulfide $\left(\mathrm{Na}_{2} \mathrm{~S}\right)$ based on the reported procedure [13]. 15DCAQ and $\mathrm{Na}_{2} \mathrm{~S}$ were reacted at $120{ }^{\circ} \mathrm{C}$ for $24 \mathrm{~h}$ in 1-methyl-2-pyrrolidone (NMP). The product was then filtered and washed with acetone and pure water several times. After drying under vacuum at $120{ }^{\circ} \mathrm{C}$, reddish PAQS powder was obtained. GSs were prepared from graphite through oxidation with $\mathrm{KClO}_{3}$ and fuming nitric acid followed by rapid thermal exfoliation at $1050{ }^{\circ} \mathrm{C}$ for $15 \mathrm{~s}$ in a muffle furnace [46,47]. To prepare the composite of PAQS and GSs (PAQS/GSs), GSs were dispersed in NMP by ultrasonication before adding 15DCAQ and $\mathrm{Na}_{2} \mathrm{~S}$. The influence of in situ polymerization was investigated through comparison with the mechanically mixed PAQS and GSs (PAQS/GSs(m)). To prepare PAQS/GSs(m), PAQS and GSs were dispersed in acetone by ultrasonication. The acetone was evaporated and PAQS/GSs $(\mathrm{m})$ was obtained. The weight ratio of PAQS to GSs was fixed at 6:4 for all of the samples in this study.

\subsection{Characterization}

Fourier transform infrared spectroscopy (FT-IR) spectra of the prepared PAQS, GSs and PAQS/GSs were recorded on an IRPrestige-21 spectrometer (Shimadzu Corp., Kyoto, Japan). Raman spectra of the samples were recorded on an NR1800 (Jasco Corp., Tokyo, Japan) using a $514.5 \mathrm{~nm}$ argon-ion laser. The crystal structures of PAQS, GSs and PAQS/GSs composite were confirmed by means of X-ray diffraction (XRD) analysis using a Bruker D8 ADVANCE diffractometer (Bruker, Billerica, USA). The Brunauer-Emmett-Teller (BET) surface areas of the samples were measured using a TriStar 3000 (Micromeritics, Norcross, GA, USA). The morphology of GSs, PAQS/GSs(m) and PAQS/GSs was observed by scanning electron microscopy (SEM) on an SU8000 (Hitachi, Tokyo, Japan) and transmission electron microscopy (TEM) on an H-9000NAR (Hitachi, Tokyo, Japan).

\subsection{Electrochemical Measurement}

Electrochemical measurement was performed using a three-electrode cell containing $0.5 \mathrm{M} \mathrm{H}_{2} \mathrm{SO}_{4}$ aqueous solution as the electrolyte. $\mathrm{An} \mathrm{Ag} / \mathrm{AgCl}$ electrode and $\mathrm{Pt}$ mesh were used as the reference and 
the counter electrode, respectively. The working electrode was fabricated by mixing active material (PAQS/GSs, PAQS/GSs(m), GSs), Ketjen black and PTFE (polytetrafluoroethylene) with a weight ratio of 6:3:1 and pressing the mixture onto a Ti mesh under a loading of $5 \mathrm{mg} \cdot \mathrm{cm}^{-2}$ at a pressure of $\sim 300 \mathrm{MPa}$. Cyclic voltammetry tests and galvanostatic charge/discharge tests were carried out using a VMP3 (BioLogic, Grenoble, France).

\section{Conclusions}

Poly(anthraquinonyl sulfide) (PAQS)/graphene sheets (GSs) composite was developed as an electrode material for electrochemical capacitors using $0.5 \mathrm{M} \mathrm{H}_{2} \mathrm{SO}_{4}$ aqueous solution. PAQS was uniformly dispersed on the surface of GSs through simple in situ polymerization. PAQS/GSs showed a pair of strong redox peaks at around $0 \mathrm{~V}$ and good cycling stability. The specific capacitance of PAQS/GSs was $349 \mathrm{~F} \cdot \mathrm{g}^{-1}$ at a current density of $500 \mathrm{~mA} \cdot \mathrm{g}^{-1}$, and a capacitance of $305 \mathrm{~F} \cdot \mathrm{g}^{-1}$ was maintained even at a high current density of $5000 \mathrm{~mA} \cdot \mathrm{g}^{-1}$. The superior electrode properties of PAQS/GSs are attributed to the fast charge transfer and redox reaction resulting from the introduction of GSs in the composite.

\section{Acknowledgments}

We express our appreciation to Masataka Ohgaki, Hitachi High-Tech Science Corp., for the valuable discussions on the composite structure.

\section{Author Contributions}

Conceived and designed the experiments: Wonkyun Lee, Shinya Suzuki and Masaru Miyayama. Performed the experiments: Wonkyun Lee. Analyzed the data: Wonkyun Lee, Shinya Suzuki and Masaru Miyayama. Wrote the paper: Wonkyun Lee, Shinya Suzuki and Masaru Miyayama.

\section{Conflicts of Interest}

The authors declare no conflict of interest.

\section{References}

1. Conway, B.E. Transition from "Supercapacitor" to "Battery" behavior in electrochemical energy storage. J. Electrochem. Soc. 1991, 138, 1539-1548.

2. Burke, A. Ultracapacitors: Why, how, and where is the technology. J. Power Sources 2000, 91, $37-50$.

3. Nishino, A. Capacitors: Operating principles, current market and technical trends. J. Power Sources 1996, 60, 137-147.

4. Simon, P.; Gogotsi, Y. Materials for electrochemical capacitors. Nat. Mater. 2008, 7, 845-854.

5. Zheng, J.P.; Huang, J.; Jow, T.R. The limitations of energy density for electrochemical capacitors. J. Electrochem. Soc. 1997, 144, 2026-2031.

6. Sarangapani, S.; Tilak, B.V.; Chen, C.-P. Materials for electrochemical capacitors. J. Electrochem. Soc. 1996, 143, 3791-3799. 
7. Zheng, J.P.; Jow, T.R. A new charge storage mechanism for electrochemical capacitors. J. Electrochem. Soc. 1995, 142, L6-L8.

8. Lokhande, C.D.; Dubal, D.P.; Joo, O.S. Metal oxide thin film based supercapacitors. Appl. Phys. 2011, 11, 255-270.

9. Naoi, K.; Ishimoto, S.; Ogihara, N.; Nakagawa, Y.; Hatta, S. Encapsulation of nanodot ruthenium oxide into KB for electrochemical capacitors. J. Electrochem. Soc. 2009, 156, A52-A59.

10. Snook, G.A.; Kao, P.; Best, A.S. Conducting-polymer-based supercapacitor devices and electrodes. J. Power Sources 2011, 196, 1-12.

11. Lu, X.; Zhang, W.; Wang, C.; Wen, T.C.; Wei, Y. One-dimensional conducting polymer nanocomposites: Synthesis, properties and applications. Prog. Polym. Sci. 2011, 36, 671-712.

12. Long, Y.Z.; Li, M.M.; Gu, C.; Wan, M.; Duvail, J.L.; Liu, Z.; Fan, Z. Recent advances in synthesis, physical properties and applications of conducting polymer nanotubes and nanofibers. Prog. Polym. Sci. 2011, 36, 1415-1442.

13. Song, Z.; Zhan, H.; Zhou, Y. Anthraquinone based polymer as high performance cathode material for rechargeable lithium batteries. Chem. Commun. 2009, 448-450.

14. Liang, Y.; Tao, Z.; Chen, J. Organic electrode materials for rechargeable lithium batteries. Adv. Energy Mater. 2012, 2, 742-769.

15. Hanyu, Y.; Ganbe, Y.; Honma, I. Application of quinonic cathode compounds for quasi-solid lithium batteries. J. Power Sources 2013, 221, 186-190.

16. Senoh, H.; Yao, M.; Sakaebe, H.; Yasuda, K.; Siroma, Z. A two-compartment cell for using soluble benzoquinone derivatives as active materials in lithium secondary batteries. Electrochim. Acta 2011, 56, 10145-10150.

17. Yao, M.; Yamazaki, S.; Senoh, H.; Sakai, T.; Kiyobayashi, T. Crystalline polycyclic quinone derivatives as organic positive-electrode materials for use in rechargeable lithium batteries. Mat. Sci. Eng. B. 2012, 177, 483-487.

18. Wang, W.; Xu, W.; Cosimbescu, L.; Choi, D.; Li, L.; Yang, Z. Anthraquinone with tailored structure for a nonaqueous metal-organic redox flow battery. Chem. Commun. 2012, 48, 6669-6671.

19. Choi, W.; Harada, D.; Oyaizu, K.; Nishide, H. Aqueous electrochemistry of poly(vinylanthraquinone) for anode-active materials in high-density and rechargeable polymer/air batteries. J. Am. Chem. Soc. 2011, 133, 19839-19843.

20. Kalinathan, K.; DesRoches, D.P.; Liu, X.; Pickup, P.G. Anthraquinone modified carbon fabric supercapacitors with improved energy and power densities. J. Power Sources 2008, 181, 182-185.

21. Roldan, S.; Blanco, C.; Granda, M.; Menendez, R.; Santamaria, R. Towards a further generation of high-energy carbon-based capacitors by using redox-active electrolytes. Angew. Chem. Int. Ed. 2011, 50, 1699-1701.

22. Tomai, T.; Mitani, S.; Komatsu, D.; Kawaguchi, Y.; Honma, I. Metal-free aqueous redox capacitor via proton rocking-chair system in an organic-based couple. Sci. Rep. 2014, 4, 3591.

23. Gall, T.L.; Reiman, K.H.; Grossel, M.C.; Owen, J.R. Poly(2,5-dihydroxy-1,4-benzoquinone-3,6methylene): A new organic polymer as positive electrode material for rechargeable lithium batteries. J. Power Sources 2003, 119, 316-320.

24. Liu, K.; Zheng, J.; Zhong, G.; Yang, Y. Poly(2,5-dihydroxy-1,4-benzoquinonyl sulfide) (PDBS) as a cathode material for lithium ion batteries. J. Mater. Chem. 2011, 21, 4125-4131. 
25. Xu, W.; Read, A.; Koech, P.K.; Hu, D.; Wang, C.; Xiao, J.; Padmaperuma, A.B.; Graff, G.L.; Liu, J.; Zhang, J. Factors affecting the battery performance of anthraquinone-based organic cathode materials. J. Mater. Chem. 2012, 22, 4032-4039.

26. Luo, B.; Liu, S.; Zhi, L. Chemical approaches toward graphene-based nanomaterials and their applications in energy-related areas. Small 2012, 8, 630-646.

27. Brownson, D.A.C.; Kampouris, D.K.; Banks, C.E. An overview of graphene in energy production and storage applications. J. Power Sources 2011, 196, 4873-4885.

28. Gómez-Navarro, C.; Weitz, R.T.; Bittner, A.M.; Scolari, M.; Mews, A.; Burghard, M.; Kern, K. Electronic transport properties of individual chemically reduced graphene oxide sheets. Nano Lett. 2007, 7, 3499-3503.

29. Stankovich, S.; Dikin, D.A.; Dommett, G.H.B.; Kohlhaas, K.M.; Zimney, E.J.; Stach, E.A.; Piner, R.D.; Nguyen, S.T.; Ruoff, R.S. Graphene-based composite materials. Nature 2006, 442, 282-286.

30. Song, Z.; Xu, T.; Gordin, M.L.; Jiang, Y.; Bae, I.; Xiao, Q.; Zhan, H.; Liu, J.; Wang, D. Polymer-graphene nanocomposites as ultrafast-charge and -discharge cathodes for rechargeable lithium batteries. Nano Lett. 2012, 12, 2205-2211.

31. Zhang, J.; Zhao, X.S. Conducting polymers directly coated on reduced graphene oxide sheets as high-performance supercapacitor electrodes. J. Phys. Chem. C 2012, 116, 5420-5426.

32. Luo, Y.; Kong, D.; Jia, Y.; Luo, J.; Lu, Y.; Zhang, D.; Qiu, K.; Li, C.M.; Yu, T. Self-assembled graphene@PANI nanoworm composites with enhanced supercapacitor performance. RSC Adv. 2013, 3, 5851-5859.

33. Chen, W.; Yan, L.; Bangal, P.R. Chemical reduction of graphene oxide to graphene by sulfur-containing compounds. J. Phys. Chem. C 2010, 114, 19885-19890.

34. Deng, W.; Liang, X.; Wu, X.; Qian, J.; Cao, Y.; Ai, X.; Feng, J.; Yang, H. A low cost, all-organic Na-ion battery based on polymeric cathode and anode. Sci. Rep. 2013, 3, 2671.

35. Ferrari, A.C.; Robertson, J. Interpretation of Raman spectra of disordered and amorphous carbon. J. Phys. Rev. B 2000, 61, 14095-14107.

36. Kudin, K.N.; Ozbas, B.; Schniepp, H.C.; Prud'homme, R.K.; Aksay, I.A.; Car, R. Raman spectra of graphite oxide and functionalized graphene sheets. Nano Lett. 2008, 8, 36-41.

37. Piaggio, P.; Cuniberti, C.; Dellepiane, G.; Campani, E.; Gorini, G.; Masetti, G.; Novi, M.; Petrillo, G. Vibrational spectra and assignment of poly-(p-phenylene sulfide) and its oligomers. Spectrochim. Acta 1989, 45, 347-356.

38. Canamares, M.V.; Garcia-Ramos, J.V.; Domingo, C.; Sanchez-Cortes, S. Surface-enhanced Raman scattering study of the adsorption of the anthraquinone pigment alizarin on $\mathrm{Ag}$ nanoparticles. J. Raman Spectrosc. 2004, 35, 921-927.

39. Yan, J.; Wei, T.; Shao, B.; Fan, Z.; Qian, W.; Zhang, M.; Wei, F. Preparation of a graphene nanosheet/polyaniline composite with high specific capacitance. Carbon 2010, 48, 487-493.

40. Wang, D.; Li, F.; Zhao, J.; Ren, W.; Chen, Z.; Tan, J.; Wu, Z.; Gentle, I.; Lu, G.Q.; Cheng, H. Fabrication of graphene/polyaniline composite paper via in situ anodic electropolymerization for high-performance flexible electrode. ACS Nano 2009, 3, 1745-1752. 
41. Yan, X.; Chen, J.; Yang, J.; Xue, Q.; Miele, P. Fabrication of free-standing, electrochemically active, and biocompatible graphene oxide-polyaniline and graphene-polyaniline hybrid papers. ACS Appl. Mater. Interfaces 2010, 2, 2521-2529.

42. Nokami, T.; Matsuo, T.; Inatomi, Y.; Hojo, N.; Tsukagoshi, T.; Yoshizawa, H.; Shimizu, A.; Kuramoto, H.; Komae, K.; Tsuyama, H.; et al. Polymer-bound pyrene-4,5,9,10-tetraone for fast-charge and-discharge lithium-ion batteries with high capacity. J. Am. Chem. Soc. 2012, 134, 19694-19700.

43. Zhao, L.; Wang, W.; Wang, A.; Yuan, K.; Chen, S.; Yang, Y. A novel polyquinone cathode material for rechargeable lithium batteries. J. Power Sources 2013, 233, $23-27$.

44. Song, Z.; Zhan, H.; Zhou, Y. Polyimides: Promising energy-storage materials. Angew. Chem. Int. Ed. 2010, 49, 8444-8448.

45. Song, Z; Zhou, H. Towards sustainable and versatile energy storage devices: An overview of organic electrode materials. Energy Environ. Sci. 2013, 6, 2280-2301.

46. Brodie, B.C. Sur le poids atomique du graphite. Ann. Chim. Phys. 1860, 59, 466-472.

47. Chen, G.; Weng, W.; Wu, D.; Wu, C.; Lu, J.; Wang, P.; Chen, X. Preparation and characterization of graphite nanosheets from ultrasonic powdering technique. Carbon 2004, 42, 753-759.

(C) 2014 by the authors; licensee MDPI, Basel, Switzerland. This article is an open access article distributed under the terms and conditions of the Creative Commons Attribution license (http://creativecommons.org/licenses/by/3.0/). 